\title{
Research on Construction of Regulatory Sandbox for Safety Supervision on Intelligent Connected Products
}

\author{
Yongqin Feng ${ }^{1, \mathrm{a}}$, Le Liu ${ }^{1, \mathrm{~b}}$,Wenzhao $\mathrm{Li}^{1, \mathrm{c}}$ \\ ${ }^{1}$ Product Safety Research Institute, China National Institute of Standardization, Beijing, China
}

\begin{abstract}
In the context of the explosive development of the global intelligent connected industry, the implementation of inclusive and prudential supervision on intelligent connected products may help to maximize the dividends of innovation-driven development strategies. As an innovation of the supervision system by government departments in the process of balancing innovation and risk, regulatory sandbox provides a forward-looking supervision mode. This paper studies and proposes the ideas for construction of China's regulatory sandbox for safety supervision on intelligent connected products by analyzing the rise, creation and role of regulatory sandbox as well as the challenges faced in the course of safety supervision on intelligent connected products.
\end{abstract}

\section{Introduction}

With the rise of China's dominant position in economic globalization in recent years, "Buy globally, Sell globally" has brought new technologies, new products and new formats to the market. The large-scale application of new technologies such as artificial intelligence and the Internet of Things has led to a rapid growth in the market for intelligent connected products, such as smart cars, unmanned aerial vehicles, smart home devices, wearable devices and smart toys. According to a joint survey conducted by Machina, IMS and Huawei, the number of connected vehicles in the world is about 90 million in 2018 and it is expected to increase to about 300 million by 2020 and exceed 1 billion by 2025[1]. Under this background, exploring the product safety supervision mode that matches the intelligent connected products is not only conducive to improving the quality and safety of products, but also helping to maximize the dividends of innovationdriven development strategies. Therefore, it has become a key issue and practical need to the current product safety supervision in China.

\section{Generation, Creation and Role of Regulatory Sandbox}

\subsection{Generation of regulatory sandbox}

The term "Regulatory Sandbox" refers to a regulatory model that allows innovators to test new products, new services and new business models by breaking through individual rules under the existing regulatory framework.

The regulatory sandbox was firstly introduced into the financial regulatory field by the UK. In 2015 , the UK
Financial Conduct Authority (FCA) officially established the FinTech Regulatory Sandbox and opened the first regulatory sandbox in May 2016. 18 enterprises from more than 60 fintech companies and traditional financial companies that make application were selected to be incorporated in the regulatory sandbox. Subsequently, Singapore, South Korea, Germany, the United States and other countries have launched their own regulatory sandboxes. The regulatory sandbox is widely used in the fields of finance, health, transportation, aviation and energy etc.

\subsection{Creation of regulatory sandbox}

The logic for creating regulatory sandbox is to achieve industrial innovation and allow breakthrough innovations to the certain extent[2]. The regulatory sandbox has main features as follows: Firstly, it aims to provide a safe test environment and supervision test area for cutting-edge innovations, similar to the pilot demonstration in China; Secondly, it focuses on substantial innovations in the sandbox access standards and emphasize full exposure of risks and protection of consumers' interests in the sandbox testing process; Thirdly, the regulatory sandbox has a complete feedback mechanism to give quick feedback on whether the innovation is applicable for formal promotion by testing the innovation in a real-time market or controlled environment for a limited time.

The process of creating a regulatory sandbox is as follows: Firstly, the government regulatory authority establishes a regulatory sandbox access standard and enterprises apply for access to the standard; secondly, the government regulatory department determines the enterprises to be incorporated in regulatory sandbox through evaluation; thirdly, on the premise of protecting the rights and interests of consumers, the enterprises are

afengyq@dpac.org.cn bliule@dpac.gov.cn cliwzh@dpac.gov.cn 
subject to the test on their innovative products, services and models; Fourthly, the government regulatory department monitors the test process and evaluates the test to determine whether it is promoted outside the sandbox.

\subsection{Role of regulatory sandbox}

Firstly, it is conducive to enhancing the inclusiveness of regulatory policies[3]. The creation of regulatory sandbox has enabled the regulatory agencies to establish closer links with innovation-based enterprises, promoted the communication and exchange between regulatory agencies and market participants, helped to identify innovation-related risks and its impact on regulatory policies and provided a reference for the formulation and revision of regulatory policies, so as to enable regulatory agencies to update those regulations that may prohibit beneficial innovation. In this way, regulatory sandbox promotes the improvement of policy environment, greatly enhances the inclusiveness of regulatory policies and resolves the contradiction and conflict incurred between the flexibility of innovation and rigidity of regulation.

Secondly, it is conducive to promoting sufficient competition in the market. The regulatory sandbox simplifies the authorization process to shorten the time to market, reduces regulatory uncertainty and facilitates the financing of innovation-based enterprises, so as to reduce the barriers to access for innovation-based enterprises and promote the access and development of new enterprises in the market. The new enterprises, in turn, may strengthen the full competition in the market, so as to produce a wider industry chain reaction and made the market full of vitality.

Thirdly, it is conducive to fully protecting the interests of consumers. The regulatory sandbox is a way for regulatory agencies to support innovation while ensuring full protection of consumers. Relying on early participation in new products, new services and new business models, regulatory agencies understand the potential problems and risks of innovation and provide comprehensive recommendations on consumer protection requirements to innovation-based enterprises, so as to promote compliance innovation, provide consumers with more and better products for choice and enable consumers to get more types of products with better after-sales products at lower prices by ensuring the protection of consumer interests.

\section{Practice of regulatory sandbox in the field of product safety}

At present, although there are different terms for regulatory sandbox in different countries and regions, such as innovation space, regulatory test bed, living labs or real life experiments are often used for regulatory sandbox and similar tests, regulatory sandbox has become a synonym for regulatory innovation. OECD points out that regulatory sandbox is particularly suitable for innovation based on digital technology and is one of the important methods to promote the application of digital related innovation[4]. Among them, the application of regulatory sandbox in the field of product safety mainly includes the following practices.

Delivery Robot Pilot. In 2016, the Hamburg Economic, Transportation and Innovation Authority launched a delivery robot pilot project, which allowed starship technologies to test delivery robots on the streets of Hamburg. The scope of the test was limited to an area of 3 kilometers around the hermes parcel shop in the suburbs of Volksdorf, Harvestehude and Ottensen. The test period is from September 9, 2016 to March 31, 2017. Daily delivery time is limited to 9 a.m. to 5 p.m. It is only allowed to deploy vehicles on public streets from sunrise to sunset, but not in twilight, darkness or poor visibility or heavy fog and rain. Based on the results of the delivery robot test, a proposal on the preconditions for authorizing automatic delivery robots in public transport was submitted to the joint meeting of the director general for transport and road construction and was accepted and forwarded to the Federal Ministry of Transport and Digital Infrastructure. The Department then asked the federal highway institute to identify legal barriers to the operation of unaccompanied delivery robots.

The Automatic Driving Vehicle Testing Platform (Kcity). In November 2016, South Korea allowed self driving vehicles to run nationwide. In order to ensure the safety of automatic driving and realize the repeated testing of autopilot vehicles in various scenes and road environments, South Korea has established the world's largest automated driving vehicle testing platform, Kcity[5]. K-city is mainly built on the intelligent transportation system of Korea traffic safety bureau. Automatic driving vehicle can be tested after obtaining temporary government permission. SK Telecom, Naver, Samsung Electronics, as well as modern automobile companies and KIA motor manufacturers will gather in Kcity, through continuous testing to improve the technology and services of self driving cars.

Digital Test Platform for Auto Driving. In order to promote the development of innovative technologies such as automatic driving, in September 2017, the Federal Ministry of Transport and Digital Infrastructure of Germany established a digital test platform for automatic driving[6]. The platform provides an innovation testing field for automobile manufacturers, parts supply industry, telecommunication companies and research institutions, which is used for innovation testing of forward-looking systems and technologies such as automation and automatic driving. The practical data obtained from the experiment in different complex environments can provide a reference for the government to improve relevant regulatory policies.

Unmanned Aircraft Systems (UAS) Pilot. In response to the rapid development of UAVs, in May 2018, the U.S. Department of Transportation selected 10 state, local and Tribal governments as participants in the pilot project of unmanned aerial vehicle system (UAS) integration, which was specifically implemented by the Federal Aviation Administration. By the end of 2020, the project will collect data on UAV night operations, package delivery, detection and avoidance technology, as well as reliability and safety of UAV data connection, so as to help the FAA to build new regulation and provide support for UAV to be included in national airspace regulation [7]. 
In addition, the UK Office of gas and electricity markets tested the business model derived from digital innovations such as smart meters through the regulatory sandbox to demonstrate whether the current regulatory model has problems and whether it should be adjusted. The U.S. Food and Drug Administration (FDA) adopts the sandbox model to establish a digital health innovation action plan. Through the launch of innovation pilot, the development of medical software pre certification for digital medical technology supervision. The Ministry of Health of Singapore (MOH) supervises the sandbox through the design of Leap design license and adaptation plan programs, so that innovative healthcare models and services can be developed and improved in a safe and controllable environment [8].

\section{Necessity of establishing the regulatory sandbox for Safety Supervision in China}

\subsection{Data analysis of product safety supervision in China}

Defective product recall is an internationally product safety supervision system. The data of defective product recall reflect the general situation of product safety in a country.

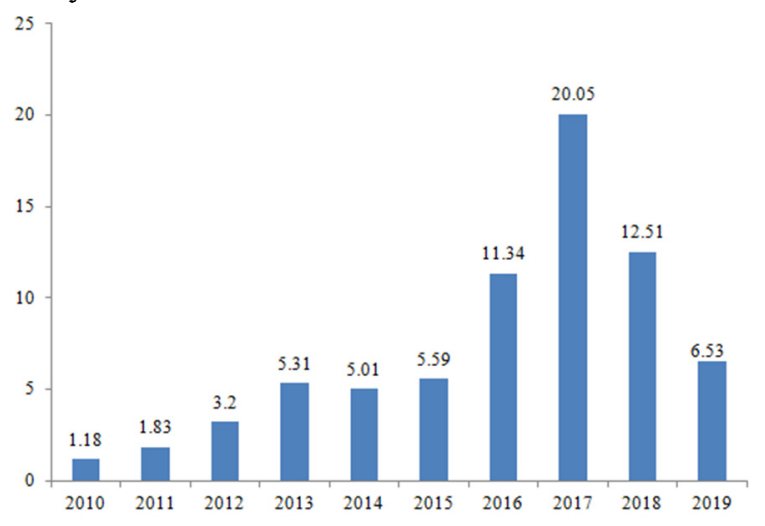

Fig1. Volume of automobile recalls in 2010-2019(million).

Since the establishment of defective automobile recall system in China in 2004, great progress has been made in automobile recall. As is shown in Fig. 1, the volume of automobile recalls in China from 2010 to 2019 is on the rise. After the promulgation and implementation of the Regulation on the Administration of Defective Automobile Products Recall in 2013, the volume of annual recalls exceeded 5 million for three consecutive years; after the promulgation and implementation of the Implementing Measures for the Regulations on the Administration of Recall of Defective Automobile Products in 2016, the volume of annual recalls exceeded 10 million for the first time; in 2017, affected by the Takata airbag problem, the volume of recalls exceeded 20 million.

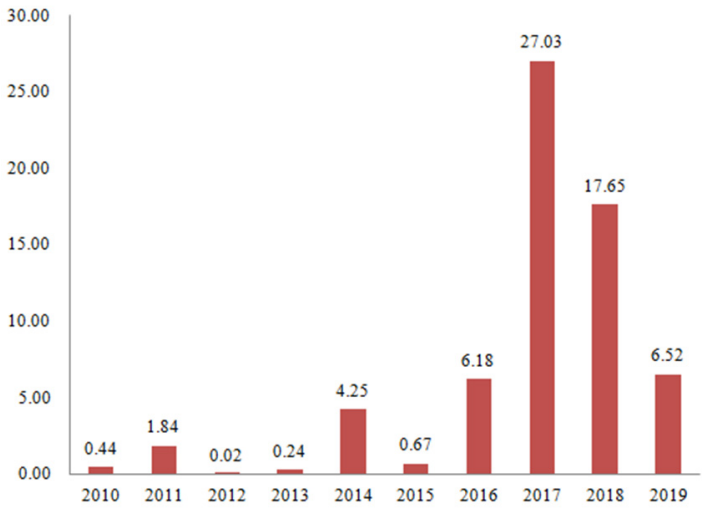

Fig2. Volume of consumer product recalls in 20102019(million)

China's consumer products recall system was established in 2017. Since 2010, the volume of consumer product recalls have been increasing year by year. As is shown in Fig. 2, 2016 was the first year of the implementation of the Measures for Administration of Recall of Defective Consumer Products, and the annual recall number exceeded 5 million for the first time. In 2017 , the number of recalls exceeded 20 million.

\subsection{New challenges faced by China's product safety supervision}

From a product safety perspective, intelligent connected products such as smart cars, unmanned aerial vehicles, smart home devices, wearable devices and smart toys allow consumers to access and use them remotely, providing consumers with more convenient and efficient services. However, due to these technical features of the above products, they also bring many potential safety risks to consumers, such as the loss of safety function due to defects, software upgrades or connection losses and personal injury to consumer due to damage of data supporting safety function. Smart connected products are the important frontier innovation driven by a new round of technology development. They not only bring profound changes to products, services, sales channels and business models, but also make new challenges to existing laws and regulations on product safety as well as existing regulatory systems. They are mainly manifested in three aspects as follows:

Firstly, product safety risks present a new model. Intelligent connected products are operated through Internet connection, so they are possibly exposed to digital security risks, such as remote manipulation and malicious tampering. For example, in 2015, hackers wirelessly invaded a CAN bus network system of a running JEEP Cherokee SUV, causing the car to fall over. In July 2015, Fiat Chrysler announced a voluntary recall of 1.4 million cars, trucks and SUVs, because those vehicles were subject to a software failure that made hackers remotely control the brakes and other systems.

Secondly, product safety status is unstable. The products that are deemed as safe when they are put on the market may become unsafe due to software upgrades or product remote cracking and changes in the product code, which makes product safety supervision involve the entire process of product life cycle. For example, after Tesla's 
over-the-air upgrade (OTA) of Model 3 in 2018, those vehicles lost key safety functions (including automatic emergency braking and Autopilot) only after more than a day and were not restored until a new update was released.

Thirdly, the hazards of product safety issues have expanded. For intelligent connected products, due to the interconnection among devices in the Internet of Things, product safety issues may be amplified and more harmful. For example, Internet of Vehicles data contains the information on roads and geography, which are closely related to national security. Automobile network services and background services may be provided by overseas communication companies and vehicle manufacturers. In this case, national geographic location information may be disclosed and national security may be endangered when communication data and Internet of Vehicle data are transmitted overseas.

In the context of the explosive development of the global intelligent connected industry, the trend of intelligent, connected and digitalized intelligent connected products has become increasingly prominent. The safety issues of intelligent connected products are not only related to the safety of consumers' own property, but also related to a "big safety" of social and national security. If the regulatory agencies fail to provide timely feedback on the cutting-edge market innovation of intelligent connected products, it may expand the externality of corporate innovation risk and increase innovation-related cost or lead to the failure of supervision, capture of supervision and coexistence of supervision and rentseeking, causing serious economic risks and social risks.

\section{Construction of regulatory sandbox for intelligent connected products in China}

The research on the generation, creation and role of regulatory sandboxes shows that regulatory sandbox is an institutional innovation undertaken by regulatory agencies in response to a new round of technological change led by digital technologies such as big data, Internet of Things and artificial intelligence, which fully embodies the regulatory agencies' cautious attitude towards innovation risk. This approach provides ideas for China to cope with the new challenges of product safety supervision brought by intelligent connected products.

Firstly, put sandbox mechanism in trial and optimize the supervision system. According to the theory of regulatory sandbox, explore the establishment of regulatory sandbox for safety of the Internet of Things, intelligent products and other new technologies and new products safety regulatory sandbox, optimize product safety supervision system, reduce the game against supervision rules, improve supervision efficiency and reduce compliance costs. At the same time, apply the regulatory sandbox concept, include new areas such as Vehicle Emission-related Recall, unmanned aerial vehicles and wearable devices into the scope of recalls by issuing announcements or guidelines, and strengthen the management on recalls through administrative means such as recall, information disclosure and penalties.
Secondly, strengthen risk prevention and control and protect consumers' interests and rights. Product safety is closely related to consumer interests and rights. However, at present, many new technologies and new products are not mature in the market, which makes consumers exposed to higher risks. Therefore, the creation of regulatory sandbox for safety supervision on intelligent connected products should focus on strengthening the prevention and control of safety risks, such as establishing a timely feedback mechanism and specific compensation standards for potential risks that are possibly faced by consumers, conducting assessment on the safety risks of intelligent connected products and claiming insurance companies to underwrite etc.

Thirdly, pay attention to evaluation feedback and establish a closed-loop mechanism. The regulatory sandbox not only encourages innovation, but also provides a window of opportunity for improving laws and regulations. This means that it is necessary to discover the legal obstacles that hinder innovation in the process of implementing the regulatory sandbox for safety supervision on intelligent connected products. Depending on the testing, evaluation and feedback of the regulatory sandbox, timely find out the specific nodes that hinder innovation and put forward the suggestions for the revision and improvement of existing laws, regulations and standard systems related to product safety. The establishment of this closed-loop mechanism can fundamentally overcome the legal obstacles that hinder technological innovation of intelligent connected products.

\section{Conclusions}

The rapid development of intelligent connected products has brought great convenience to consumers. But at the same time, it has also shown new features such as unstable product safety and expansion of product safety problems, which has made new challenges to safety supervision on intelligent connected products in China. As an innovation to supervision system by government departments in the process of balancing innovation and risk, regulatory sandbox provides a responsive and forward-looking supervision mode. This paper proposes to trial the sandbox mechanism in the field of intelligent connected products and optimize the supervision system. It is noted that the establishment of regulatory sandbox for intelligent connected products is not to relax supervision or reduce safety standards, but to put forward the suggestions for formulating or revising laws and regulations through experiments in regulatory areas where the legal regulations are unclear or uncertain. In this way, it provides a direction for China to cope with the new challenges of product safety supervision on intelligent connected products.

\section{Acknowledgment}

This work was financially supported by the Fundamental Research Funds of China National Institute of Standardization Project(No. 282020Y-7513). 


\section{References}

1. "Looking at the layout of domestic Internet of vehicles manufacturers from BAT, Huawei, three major operators and FutureMove." https://ee.ofweek.com/2019-07/ART-8320315-814030400627.html, Jul 2019.

2. R. Chai. "Foreign experience and Enlightenment of Regulatory sandbox," Law Sci, pp.27-40, Aug 2017.

3. Canbridge Centre for Alternative Finance. "Early Lessons on Regulatory Innovations to Enable Inclusive FinTech: Innovation Offices, Regulatory Sandboxes, and RegTech,” 2019.

4. OECD. "Draft concept note on the policy implications of AI on product safety," Sep 2019.

5. Research Centre for Autonomous Road Vehicles. "World's largest test bed for self-driving cars to be opened in Korea". http://recar.bme.hu/eng/ worldslargest-test-bed-for-self-driving-cars-to-be-openedin-korea/, 2018.

6. BMWi. "Digital test beds". https://www.bmvi.de/EN/Topics/Digital-Matters /Digital-Test-Beds/digital-test-beds.html, 2018.

7. United States Federal Aviation Authority. "U.S.Transportation Secretary Elaine L. Chao Announces Unmanned Aircraft Systems Integration Pilot Program Selectees". https://www.faa.gov/news/press_releases/news_ story. cfm?newsId=22755, 2018.

8. Ministry of Health of Singapore. "MOH Launches First Regulatory Sandbox to Support Development of Telemedicine." https://www.moh. gov.sg/newshighlights/details/moh-launches-first-regulatorysandbox-to-support-development-of-telemedicine, 2018. 